\title{
A Practical Application of Low Voltage DC Distribution Network Within Buildings
}

\author{
B Marah ${ }^{1}$ \\ Hoare Lea LLP. London, UK \\ bmarah@theiet.org
}

\author{
Y R Bhavanam², G A Taylor ${ }^{2}$, M K \\ Darwish $^{2}$ \\ Brunel University London. London, UK \\ y.r.bhavanam@ieee.org, \\ gareth.taylor@brunel.ac.uk \\ mohamed.darwish@brunel.ac.uk
}

\author{
A O Ekwue ${ }^{3}$ \\ Jacobs Engineering Inc., \\ Croydon,UK \\ arthur.ekwue@jacobs.com
}

\begin{abstract}
The popularity of low voltage DC distribution system has increased in many countries such as the UK due to technological advances in power electronics. This is a promising feature for the future smart-grid distribution system because of its high efficiency and reliability. Hence some of the UK engineers are looking into the possibility of rolling out low voltage DC distribution in mainly commercial and residential buildings. However, the introduction of this technology presents some practical implementation challenges. This paper investigates the possibilities, benefits, and limitations of a low voltage DC distribution system within buildings.
\end{abstract}

Index Terms-load flows, Energy storage, Future smart grid, Low voltage DC distribution network, Total harmonic distortion.

\section{INTRODUCTION}

Low Voltage (LV) DC power distribution systems within buildings is a promising technology due to various advantages such as fewer conversion stages, uninterrupted power delivery, higher efficiency and reliability compared to conventional AC systems [1]. In the 1880's loads were only found in densely populated areas, the voltage used was not economically competitive as well as the copper lines needed to transfer power from A to B. Furthermore, the $I^{2} R$ power loss limited the distance power can be transferred, Michael Faraday's law of induced current due to changing of the magnetic field solved the issue by using AC system instead of DC system [2]. The advancement of power electronics has seen the return of interest in DC distribution system and this is once again gaining popularity in many countries such as the UK.

This paper is organized into six sections: the first section presents the introduction; the second section describes how DC distribution system within buildings can facilitate the EU 2020 emissions targets; the third section discusses the energy efficiency using LVDC distribution system while discussing recent studies and consumer electronics load profile; the fourth section proposes the method used; the fifth section discusses the scenario testing and harmonic analysis caused by the LVDC converter and last section presents the conclusions.

\section{LOW VOLTAGE DC DISTRIBUTION}

Efficiency is a key measure to achieve the EU 2020 targets; therefore LVDC distribution system will play a great role in improving efficiency. LVDC distribution system is more efficient than $\mathrm{AC}$ system [3] as the $\mathrm{AC}$ system is more prone to losses when it is used to distribute power within buildings due to the individual conversion stages of consumer electronics. The three targets defined by the EU as a benchmark to be achieved by 2020 are [4]:

- Emissions: $20 \%$ reduction by 2020 compared to 1990

- Renewable: The grid energy consumption should be made of $20 \%$ renewable energy

- Energy Consumption: A 20\% reduction by using energy efficient plant equipment

With all these environmental concerns the LVDC distribution system will play a prominent role in achieving the three targets stated above. For example the power generated from photovoltaic (PV) can be directly connected to the LVDC distribution system without the need for intermediate conversion. This will not only improve efficiency but will at the same time reduce the investment cost of the PV system. LVDC distribution system has more advantages compared to AC system [5]. The removal of the conversion steps for each individual consumer electronic within buildings would decrease cost and improve efficiency. It is stated in [6] that it can be assumed that every conversion steps removed will result to $2.5 \%$ increase in efficiency. In addition, LVDC distribution system with centralised AC-DC conversion would facilitate the filtering process of harmonics within buildings. The LVDC system can be more effective when it comes to connecting renewable and low carbon energy sources to the distribution grid [7]. As most of the decentralised renewable such as PV, batteries, fuel cell and other renewable sources produce DC natively or will require intermediate conversion stages to be connected to the AC grid. Therefore, all these renewable sources can be directly connected to the grid with LVDC network in place. Eventually, this will reduce the power losses during the conversation stages and reduces capital cost of the overall system [1]. Furthermore, there is a potential of energy savings by shifting to DC appliances. In [5] it is demonstrated 
that the average energy savings of $33 \%$ can be achieved when a DC alternative is chosen. Therefore there might be little other conversion required such as smaller electronic devices with a voltage between 1.8 to $5 \mathrm{~V}$. However, there are various challenges with the LVDC system apart from DC ready available consumer electronic devices, there are also technical challenges to integrate LVDC to existing AC system such as operating and protecting the AC-DC element of the network and suitable breakers to disconnect faulty element of the LVDC system. Moreover, other challenges are the missing standards to date how to operate, protect and configure the LVDC system and standard voltages to be used. Nevertheless, this paper is subjected to the analysis of the steady state behaviour of a proposed LVDC network.

\section{ENERGY EFFICIENCY USING LOW VOLTAGE DC DISTRIBUTION}

A study on energy efficiency within buildings has revealed that buildings account for $40 \%$ of the global energy consumption [8]. Building statutory guidance such as conservation of fuel and power approved document in England [9] has helped to reduce energy consumption to match the notional building. The main aim is to use energy efficiency equipment and reduce the carbon foot print of the buildings. Introducing LVDC system in building will eliminate the multiple stages of power conversions in buildings; this will be one of the elements that would help designers and engineers to meet the EU energy consumption targets. Designers and Engineers should also select energy efficient loads for commercial and residential developments which might be categorised into the following;

- Resistive load such as heating loads

- Electronic load such as multimedia equipment

- Electromechanical loads such as pump systems, HVAC and catering equipment.

Resistive loads are more prone to RMS voltage values while electronic loads are supplied with direct current with the aid of AC-DC converters and currently most of the electromechanical loads within building are dominated with AC power supply. Studies have revealed that using variable speed control for electromechanical loads could reduce energy consumption by $20 \%$ [1]. Variable speed inverters also has intermediate DC conversion an LVDC system will eliminate the need for the intermediate conversions. There is also the emerging technology of electric vehicles which relies on electrochemical battery charged with the aid of AC-DC conversion electric changers external to the vehicle or within the vehicle [10]. Moreover, PVs are used in residential and commercial buildings to compensate carbon foot print of the building as consequence PV installations are connected to the grid. As DC power is generated by the PV installations, a conversion process from $\mathrm{DC}$ to $\mathrm{AC}$ is required and yet another additional conversion losses within the building envelope. If the entire conversion step can be reduced to a single centralised conversion stage at the HV/LV secondary substation, this will not only improve the power distribution within the building but would also reduce the capital cost of the electrical building services installation and eventually improve the efficiency of power distribution within buildings.

A literature research has revealed the study of reduction of conduction losses induced by a DC distribution system and a feasibility study for several voltage levels [1]. Another study presented schemes with house hold DC network [5] and proposed a comparison between DC and AC system for PV installation with storage to support the grid. All reviewed papers fail to address the practical application of the study undertaken into the LVDC system within buildings, in particular for residential and commercial buildings. This paper complements the existing research by investigating the possibilities, benefits, and limitations of a practical low voltage DC distribution system within buildings. The steady state analysis has been reported using power system analysis software Electrical Transient Analyzer Program (ETAP). The behavioural of LVDC system within buildings has been investigated. Simulations have been undertaken using the proposed model with embedded PV generation to the LVDC distribution network. Load flows at various points in the building were established to determine the weakest point of the network. Result obtained are presented and analysed to validate the proposed model.

The use of consumer electronics in commercial buildings and its typical consumption profile has been more less the same over the decades. However, the profile for consumer electronics is changing rapidly due to the advancement of electronics in particular the silicon microcontroller's chips and the technology to use these chips and develop advanced consumer electronics. The prices and features of these devices have increased and the usage of these within homes and offices has dramatically increased in the years. Most of the consumer electronics uses DC power, however due to the standard voltage in buildings was long established to be AC voltage, the AC voltage is converted to DC system to serve the consumer electronics. Using LVDC would prevent the need for AC-DC conversion for each device. The centralised AC-DC conversion would be required at the secondary substation from where the DC power would be distributed to residential and commercial buildings as proposed in Fig. 1.

\section{PROPOSED LVDC DISTRIBUTION NETWORK}

To investigate, analyze, and propose a practical low voltage DC system, a low voltage DC model was identified and an optimal network of low voltage DC distribution within buildings was proposed. The block diagram shown in Fig. 1 was proposed which is identical to modern day AC distribution system within commercial buildings apart from its network component been DC. The diagram is a 
representation of the proposed network in Fig. 2. LVDC distribution networks are connected in different ways; the most common connections are unipolar and bipolar connections. The main differences between the two connections are the voltage levels obtainable between its terminals and their commercial performance. The bipolar has an additional zero conductor which make it possible to connect load either between the positive conductor and the zero conductor or between the positive and negative conductors [11]. For the unipolar LVDC network connection, load may only be connected between the positive and negative terminals of the LVDC network. Furthermore, a common problem associated with the bipolar LVDC network is when the connected load to the LVDC network is not identical as the network system will be unbalanced. The unipolar problem is the increase in cost of the power electronic devices in the network due to higher voltage levels along the LVDC network. Considering the overall cost between the unipolar and bipolar LVDC system, the cost for a unipolar system is relatively low compared to the bipolar network of the same size [12] and as such the proposed network has been based on the unipolar LVDC system for its commercial advantage and simplicity.

The proposed block diagram consists of 1 MVA ground mounted transformer denoted as $\mathrm{HV} / \mathrm{LV}$ on the diagram in Fig. 1 which steps down the voltage from $\mathrm{HV}$ to $\mathrm{LV}$ supplying power to the DC lumped loads of $970 \mathrm{~kW}$. Battery banks are connected in the main LVDC switch room in the event of power failure or alternatively the battery banks might be utilised during peak hours by the network operators as a means of balancing the system between generation and demand. The proposed network consists of the secondary substation, the main LVDC switch room, battery banks and three sub-distributions in electrical risers within the building and roof PV system. The three number sub-distribution systems were selected to facilitate the simulation in the ETAP software. The LV distribution network was modelled using the Electrical Transient Analyzer Program (ETAP) software package shown in Fig. 2. The LV DC converter is represented by the ETAP AC-DC converter module with harmonic spectrum and wave form of the standard IEEE 519 equation. The standard IEEE 519 equation is a widely used method for determining the total harmonics distortion; some of the difficulties that arise with the use of the standard IEEE 519 equation is determining a demand current at the design stage [7]. This is simply because the recommended definition of demand current value can only be determined by measurements taken after the electrical installation.

The power supply for the LVDC distribution system might be achieved with the proposed single line diagram in Fig. 1. It is proposed to use an automatically controlled tapped transformer which is connected in to a closed loop control system with rectifier and the voltage regulator within the loop. The output is a controlled LVDC distribution system for commercial and residential buildings. Protection for LVDC distribution system is also a challenging phenomenon as for AC system breakers can trip the system on a zero-crossing point. However, in DC systems there is no zero-crossing point, this might be solved by generating oscillations during the period of a breakers opening as the same way for resonant converters. This might be applied with detection devices for short circuit, overload and leakage protection.

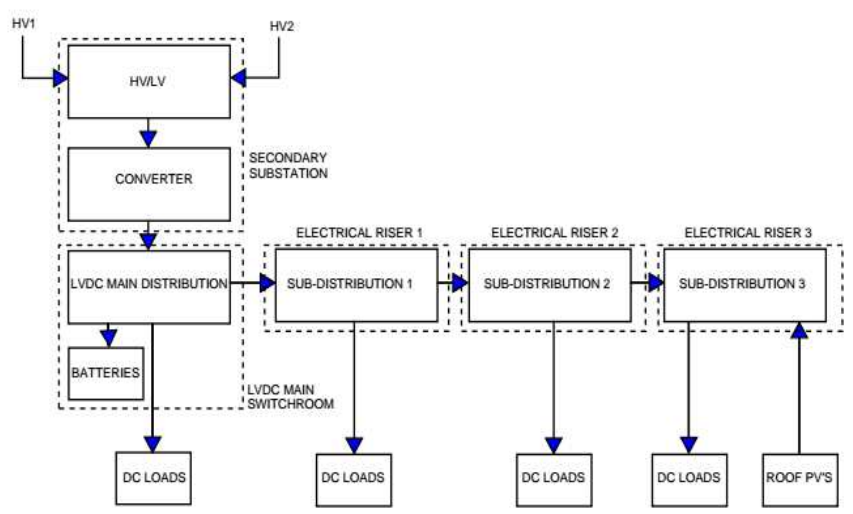

Fig. 1: Proposed LVDC diagram within commercial buildings

\section{A. Network Model}

The single line LVDC network is shown in Fig. 2; this network model has been modelled in the ETAP software for the purpose of studying the steady state behaviour of the LVDC system within buildings at different electrical subdistributions panels. The LVDC network model is assumed to be connected to a secondary $11 \mathrm{kV} / 0.4 \mathrm{kV}$ AC system with an automatic tap changer transformer, 12 pulse rectifiers and a closed loop control voltage regulator on the transformer output which is connected back to the tap changer of the transformer to ensure that voltage level at the transformer secondary voltage is always maintained. The LVDC system might provide a platform for micro-grid system. Batteries might also be connected directly and eliminate the need for any major intermediate conversion process, the LVDC network is considerably easier to operate and monitor due to the absence of the frequency component. If the power and voltages to be transmitted are similar for AC and DC, DC system is more efficient compared to its AC counterpart. As an additional bonus, the DC power line might be easily used to transmit communication and data packages which might be used to securely transmit control signal to control the network.

A feasibility study was undertaken in [1] for the use of LVDC network in commercial facilities, it concludes that the suitable DC voltage level is between $300 \mathrm{~V}$ and $400 \mathrm{~V}$ in terms of both the economical and technical points of view and for that reason and for it compatibility of three phase loads in commercial buildings a $400 \mathrm{~V}$ DC was chosen in the ETAP simulations. Therefore, the LVDC output from the 12 pulse rectifier supply is $400 \mathrm{~V}$ DC between the two poles, the 
following network parameters were used to undertake the steady state analysis;

- LV cables resistance: $0.024 \mathrm{ohm} / \mathrm{km}$

- Inductance: $0.09 \mathrm{mH} / \mathrm{km}$

- Transformer impedance: $5 \%$

- $\quad$ Ring AC voltage is: $11 \mathrm{kV}$

- Secondary voltage is $0.4 \mathrm{kV}$

The LVDC network was modelled using a 1 MVA transformer with a typical impedance of 5\% and a distribution network operator (DNO) recommended fault level of 250MVA at the ring main unit (RMU) which supplies the secondary HV/LV substation. A load about $97 \%$ of the transformer rated MVA was connected into the LVDC system at various load centre within the building, a steady state simulation was undertaken to investigate the voltage drop, current flowing through the cables, power flow and harmonics.

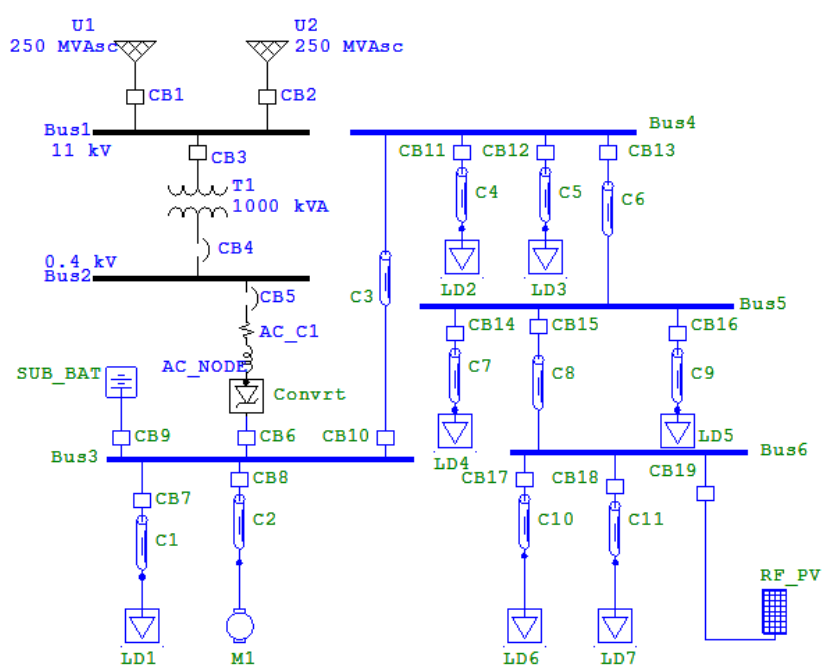

Fig. 2: Proposed LVDC distribution network within commercial buildings

\section{B. Simulation method using ETAP}

LVDC distribution network was simulated with and without the battery banks and PV system using ETAP. The assumptions for simulating the DC lump loads with and without the battery banks and PV system are as follows;

- $\quad$ The DC lump loads are an aggregated load within the building such as small power and lighting loads.

- $\quad$ The load was modelled as 8 number lump load totalling $970 \mathrm{~kW}$ at various points along the LVDC network.

- Balanced three phase load was assumed throughout the studies.

The model was simulated using ETAP software package [13]. ETAP uses the Newton Raphson, Adaptive Newton Raphson and Accelerated Gauss Seidel algorithm to solve the load flows. The Newton Raphson algorithm was chosen for the simulation because of its robustness to solve the load flows. The ETAP model provided a means of calculating not only the power flows, and the voltages for all nodes and in magnitude but it also provided the means of obtaining the complete harmonics spectrum of the AC side of the LVDC Network. The assumption of three-phase balanced system above will allow for the system to be represented by a singlephase equivalent circuit, (i.e. the positive sequence component of the network). In addition this will allow the representation of the network as a single line diagram in the ETAP software package as shown in Fig. 2.

The 8 number DC lumped loads connected on the HV/LVDC radial distribution network consist of bus-bars with supply points $\mathrm{U} 1$ and $\mathrm{U} 2$ which forms part of the ring main system as a measure of resilience. The radial network supplying the DC lump loads originates from a HV/LV substation where the $11 \mathrm{kV}$ is step-down to $0.4 \mathrm{kV}$ with the aid of $\mathrm{HV} / \mathrm{LV}$ transformer and the $0.4 \mathrm{kV}$ voltage serves as the main source for the low voltage DC network. The connected converter converts the low voltage AC to low voltage DC and two different steps were followed to analyse the network:

a) The DC lumped loads were simulated without the battery banks and PV been connected to the network and result for voltage spectrum, power flow and total harmonics distortion were observed.

b) The DC lumped loads were simulated with the battery banks and roof PV been connected to the network while the supply to the network was disconnected and result for voltage spectrum, power flow and total harmonics distortion were observed.

\section{LVDC DISTRIBUTION NETWORK ANALYSIS}

The two scenarios above were evaluated. Based on the ETAP DC and harmonic load flow simulations carried out, it is evident that the DC load behaviour on the proposed LVDC distribution network provides more accurate information regarding the LVDC feeder voltage drops and total harmonic distortions on the AC side of the network depending on the harmonic order.

A. Test Case 1: DC lumped loads are connected to the distribution network and power flowing from the secondary substation to DC loads.

In this test case a DC lumped load of $970 \mathrm{~kW}$ is connected to the proposed network. The PVs and Battery banks are not connected. As the distribution load of $970 \mathrm{~kW}$ is examine for voltage drop, power flow and total harmonics distortions.

C. Test Case 2: Power failure or load shedding initiated by the network operator was assumed. 
In this test case, DC lumped loads are connected to the proposed LVDC network, while assuming a power failure or load shedding scenario initiated by the network operator to help balance the system when it needed. Here, the battery bank and PVs are connected while the network supply was disconnected to investigate whether the battery banks and installed PVs will provide enough power to the proposed LVDC network load. On performing simulations for the two different test cases, two cases are discussed below.

(i) The voltage waveform obtained at the final $\mathrm{AC}$ bus2 where LVDC being interfaced through the AC-DC converters illustrated in Fig. 3 below.

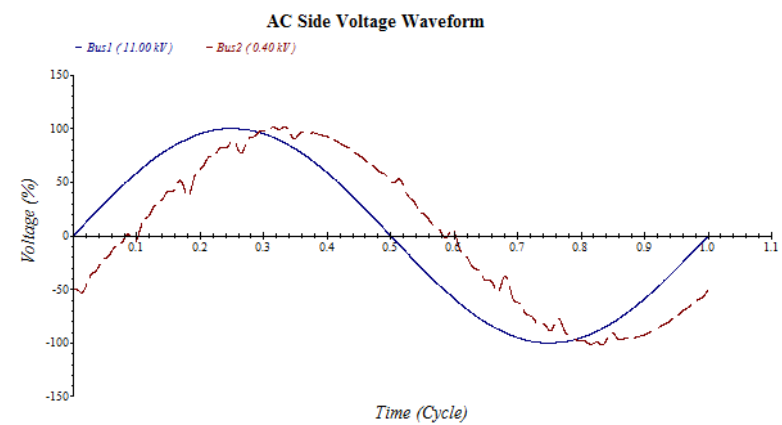

Fig.3: Voltage waveform when the DC Lumped loads are connected

The observation is also made for the current harmonics waveform at the transformer as illustrated in Fig.7. Here the third harmonic does not exist because the line-to-line voltages are the phasor difference between the line to neutral voltages and they are suppressed.

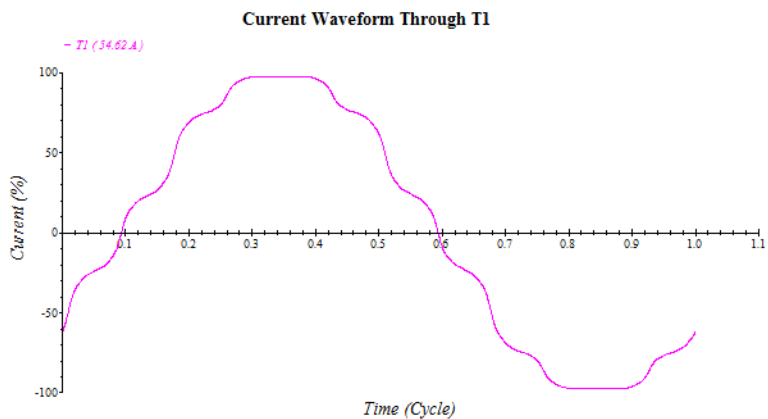

Fig. 4: Current waveform at the transformer as result of the LVDC network

The above results are obtained when the DC lumped loads are connected to the LVDC distribution network and being supplied through the grid. The voltage distortion at AC bus2 within the secondary substation is shown in Table 1 .

TABLE 1: CHANGE OF THD IN VOLTAGE (THD $)$

\begin{tabular}{|c|c|c|}
\hline Bus ID & Voltage $(\boldsymbol{k V})$ & $\boldsymbol{T H D}_{\boldsymbol{V}}(\boldsymbol{\%})$ \\
\hline 2 & 0.4 & 5.93 \\
\hline
\end{tabular}

In the IEEE recommendations for harmonic distortions [14], the total harmonic distortion in voltage can be allowed up to $5 \%$ when the voltage levels at the point of common coupling
(PCC) are $1 k V<V \leq 69 k V$. From Table 1, it can be clearly observed that the THDv exceeds the IEEE recommended limit at bus 2 where the AC-DC is interfaced with the converter. The current waveform through T1 is shown in Fig. 4 ; it is distorted as it has individual harmonic distortion in current $\left(\mathrm{IHD}_{\mathrm{i}}\right)$ of $11.72 \%$, this again exceeds the recommended limit of $10 \%$. Therefore, it is recommended to implement harmonic filters at this level to mitigate both voltage and current distortion for the LVDC network. The load flow result of the DC section and analysis downstream the AC system examined while the LVDC network is being connected to the AC grid is discussed below. In addition, a scenario where power outages or load shedding initiated by the network operator is further discussed below. The load flow result of the DC section is shown in tables and graphs. It should be noted that two scenarios of the simulations results reveal identical results and as such only the result for the first scenario has been presented here.

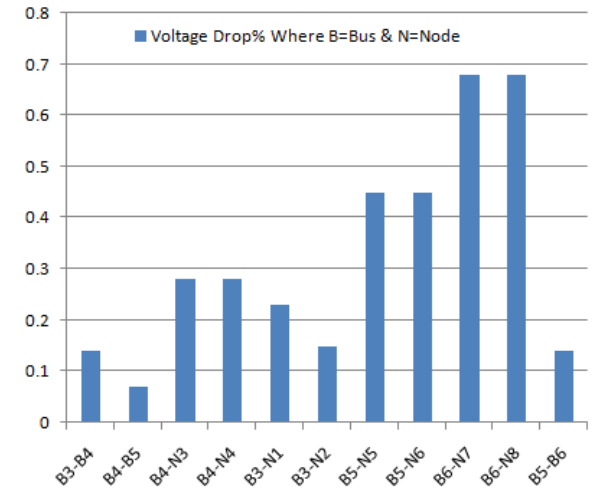

Fig. 5: Percentage of voltage drop along the LVDC network

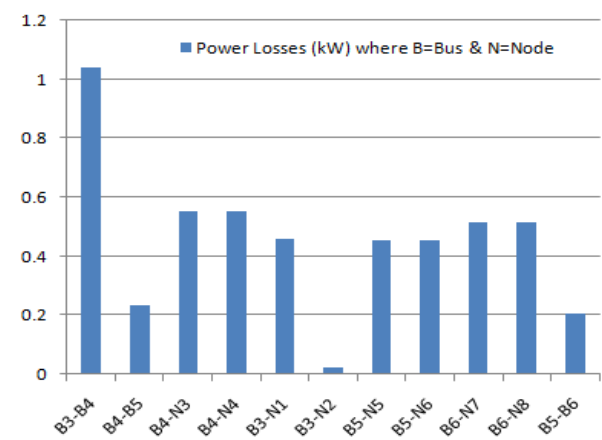

Fig. 6: Power losses in (kW) along the LVDC network

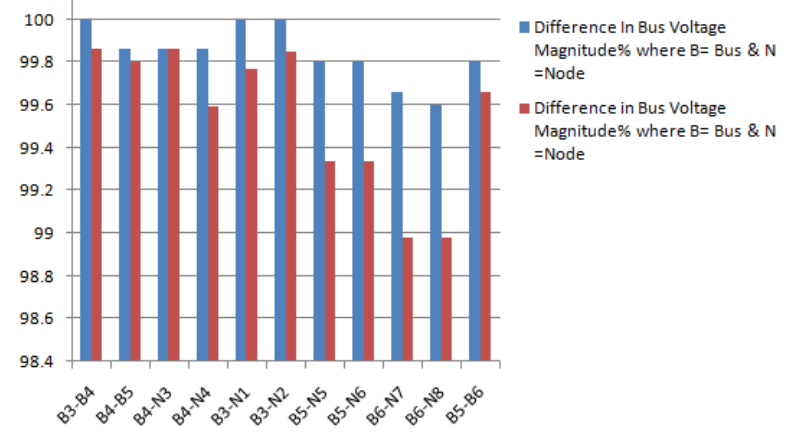

Fig. 7: Percentage of voltage drop difference along the LVDC network 
(ii) The results obtained when PVs and battery banks being switched while the network supply to the proposed LVDC network being switched off to the distribution network are identical with the result obtained in (i) and therefore has not been illustrated here.

It is evident that if batteries are sized correctly, the DC loads can be supplied during power failure or load shedding initiated by the network operator without the disruption of the building operation for an agreed time. Results for two scenarios were obtained, one was to examine the LVDC network behaviour while connected to the AC grid and the other is to examine the LVDC network while it is being fed using battery banks. Results obtained have revealed similar result in terms of power losses, voltage drop and voltage magnitude along the LVDC network. Results obtained reveal that, as this is a radial distribution network the buses which lie far away from the main radial feeder source are the most affected. This is generally true with exception of embedded generation connected downstream the LVDC network where power flows does not only flow from the radial feeder main source, but power is also being generated close to local buses within the LVDC network. Based on the analysis, it is clear that the low voltage DC distribution within building has many benefits such as (i) minimizing power loss, (ii) reducing energy usage and consequently energy savings which eventually reduce the consumers' electricity bill and the carbon footprint and (iii) improve the power quality while ensuring the economic feasibility. Results obtained have revealed that if the battery banks are sized correctly the DC loads can be supplied without disruption for a given time, the battery banks are to be sized to suit the agreed outage time.

\section{CONCLUSION}

In these studies, a low voltage DC model has been analysed and an optimal network of low voltage DC distribution within buildings has been proposed considering the nonlinearity of AC/DC conversion. At the high-voltage to low-voltage secondary substation it is recommended that harmonics filters are implemented to eliminate harmonics within the system. As the paper has demonstrated, LVDC distribution system is a promising technology within buildings. There are also challenges with LVDC distribution within buildings. For example AC building services equipments are readily available in the market, on the other hand DC ready equipment have to be custom-built which could cost as three times more than the standard products. Therefore, manufacturers should be given more incentive to develop DC ready building services and consumer electronics equipment.
It can also be concluded that low voltage DC distribution within buildings and emerging technologies towards domestic energy storage and electric vehicle battery charging will support ageing distribution networks, the storage devices can be used to support the network when required. Further study might be to investigate the transient responses of LVDC network within buildings and LVDC natural phenomena that would have impact on the behaviour of AC-DC systems. In LVDC system there are many sensitive devices based on power electronics which have their own direct impact on the characteristics of LVDC faults profile. This need to be investigated and well understood in order to facilitate the LVDC network operation and management within buildings.

\section{ACKNOWLEDGEMENTS}

The authors are grateful to Hoare Lea LLP and Brunel University for providing the facilities used for this Study.

\section{REFERENCES}

[1] A. Sannino, G. Postiglione, and M Bollen, "Feasibility of a DC network for commercial facilities.," IEEE Trans. Ind. Appl., vol. 39, pp. 1499$1507,2003$.

[2] C. L. Sulzberger, "Triumph of ac - From Pearl Street to Niagara," IEEE Power Energy Magazine, vol. 1, no. 3, pp. 64 - 67, 2003.

[3] Nuutinen et al., P, "Experiences from Use of an LVDC System in Public Electricity Distribution," in in Proc. CIRED2013 Conf., 10-13 Jun. 2013.

[4] Europenan Commission, "Summary of Directive 2010/75/EU on industrial emissions (integrated pollution prevention and control)," The Industrial Emissions Directive, 2010.

[5] Bo Normark (KIC InnoEnergy) Shivakumar A, "Household DC networks: State of the art and future prospects," An Energy Think Tank Informing The European Commission , 2015.

[6] D. J. Hammerstrom, "AC versus DC: did we get it right?," in Power Engineering Society General Meeting, 2007.

[7] George Eduful, Ebenezer A. Jackson, and Joseph Ekow Cole, "Harmonic Emission Limits and Selecting PCC Location Based on the Type of Distribution System," vol. 1, 2014.

[8] World Business Council For Sustainable Development, "Energy Efficiency In Buildings ," June 2009.

[9] HM Goverment, "Conservation Of Fuel And Power In New Dwellings: Approved Document L1A," Building Regulations , 2010.

[10] Marah. B, et al, "Impact of Electric Vehicle Charging Systems on Low Voltage Distribution Networks," in UPEC 2016/IEEE, 2016.

[11] Salonen. P, "An LVDC Distribution System Concept, " in Nordic Workshop on Power and Industrial Electronics, 2008.

[12] Kaipia.T et al, "Possibities Of The Low Voltage DC Distribution System," Lappeenranta University of Technology.

[13] ETAP, Electrical Transient Analyzer Program, 2016.

[14] IEEE std 519-2014, "IEEE Recommended Practice and Requirements for Harmonic Control in Electric Power Systems," 2014. 